\title{
Histopathology of melanosis coli and determination of its associated genes by comparative analysis of expression microarrays
}

\author{
XIAO-AN LI* ${ }^{*}$ YAN ZHOU* ${ }^{*}$ SHU-XIAN ZHOU, HAI-RONG LIU, JIN-MEI XU, \\ LONG GAO, XIAN-JING YU and XIAO-HUI LI
}

\begin{abstract}
The Gastroenterology Tumor and Microenvironment Laboratory, Department of Gastroenterology, The First Affiliated Hospital of Chengdu Medical College, Chengdu Medical College, Chengdu, Sichuan 610041, P.R. China
\end{abstract}

Received November 14, 2014; Accepted June 23, 2015

DOI: $10.3892 / \mathrm{mmr} .2015 .4126$

\begin{abstract}
Melanosis coli (MC) refers to the condition characterized by abnormal brown or black pigmentation deposits on the colonic mucosa. However, the histopathological findings and genes associated with the pathogenesis of melanosis coli remain to be fully elucidated. The present study aimed to examine the histopathological features and differentially expressed genes of MC. This involved performing hematoxylin and eosin staining, specific staining and immunohistochemistry on tissues sections, which were isolated from patients diagnosed with MC. DNA expression microarray analysis, western blotting and immunofluorescence assays were performed to analyze the differentially expressed genes of melanosis coli. The results demonstrated that the pigment deposits in MC consisted of lipofuscin. A TUNEL assay revealed that a substantial number of apoptotic cells were present within the macrophages and superficial lamina propria of the colonic epithelium. Expression microarray analysis revealed that the significantly downregulated genes were CYP3A4, CYP3A7, UGT2B11 and UGT2B15 in melanosis coli. Western blotting and immunofluorescence assays indicated that the expression of CYP3A4 in the normal tissue was higher than in the MC tissue. The results of the present study provided a comprehensive description of the histopathological characteristics and pathogenesis of MC and for the first time, to
\end{abstract}

Correspondence to: Dr Xiao-An Li, The Gastroenterology Tumor and Microenvironment Laboratory, Department of Gastroenterology, The First Affiliated Hospital of Chengdu Medical College, Chengdu Medical College, 4 Baoguang Road, Xindu, Chengdu, Sichuan 610041, P.R. China

E-mail: zqzy1983@163.com

*Contributed equally

Key words: melanosis coli, pigmentation, CYP3A4, laxatives, expression microarray the best of our knowledge, demonstrated that the cytochrome P450-associated genes were significantly downregulated in melanosis coli. This novel information can be used to assist in further investigations of melanosis coli.

\section{Introduction}

Melanosis coli (MC) is a condition, in which the mucous membrane of the colon and rectum appear darker than usual, with the depth of color varying between pale grey and brown or black (1). Billiard first described the occurrence of colonic mucosal hyperpigmentation in 1825, which Virchow termed melanosis coli in 1857. In 1928, Bartle indicated that MC was associated with long time use of laxatives, and subsequent studies investigated this association (2-4). Investigations on animal models of melanosis have indicated that anthraquinone laxatives, including aloe, senna and rhubarb cause MC $(5,6)$, however, their role in the etiology and pathogenesis of $\mathrm{MC}$ remains to be elucidated.

Several hypotheses have been suggested to explain the pigmentation of MC. The majority suggest that the formation of pigment granules is associated with purgative-induced apoptosis of colonic mucous membrane epithelial cells. The laxative effect of anthranoid laxatives induces damage on the epithelial cells; which causes alterations in absorption, secretion and motility. The outcome is harmful to the cells in the lining of the intestine and leads to apoptosis. These apoptotic cells are subsequently phagocytized by adjacent macrophages, which form a substance that appears as dark pigmentation granules (7). The distinctive pigmentation of the bowel wall develops when a sufficient number of cells have been damaged. It has also been suggested that improvements in standards of living and lack of proper exercise contribute to decreases in bowel movements and leads to chronic constipation. This, in turn, leads to an increased quantity of protein-rich foods remaining in the intestinal tract. The intestine absorbs the protein degradation products and converts them into melanin or lipofuscin by fermentation within the connective tissue cells. When melanin or lipofuscin is phagocytized by macrophages in the lamina propria, conditions are favorable for the 
development of MC $(8,9)$. Therefore, determination of whether there apoptotic cells are present in the colonic mucosa of $\mathrm{MC}$ patients is required.

The pigment bodies in the intestine may be composed of lipofuscin, melanin, hemosiderin or bile pigment, however, no clear experimental evidence has confirmed the type of pigment present in MC (10). Lipofuscin granules are residual bodies containing oxidized and/or undigested lipids. These granules are considered to result from the residue of cellular organelles within lysosomes (11). Melanin is synthesized through oxidation of tyrosine to dopamine and eventually melanin in the melanosome (12). Due to macrophage phagocytosis of erythrocytes and/or their breakdown products, hemosiderin develops within residual bodies (13). Each granule type is distinctive and can be visualized using specific staining. Confirmation of the type of pigment granules present in $\mathrm{MC}$ is required.

The presence of MC may indicate an increased risk for the development of colorectal cancer. High doses of anthraquinone cause tumor development in animals, and colorectal adenomas occur more frequently in patients with MC $(14,15)$. Therefore, MC has clinical significance, and further analysis of its clinical features and pathogenesis is necessary. In previous years, several studies have been performed to investigate $\mathrm{MC}$, however, the requirement for comprehensive investigation remains, and comparative analysis of gene expression differences in MC have not been determined. Therefore the present study aimed to investigate MC in terms of its endoscopic features, histopathological characteristic and gene expression differences, and provide a novel framework for understanding the pathogenesis of MC.

\section{Materials and methods}

Tissue and patients. A total of 26 patients with MC were recruited in the present study (Table. I), and tissue specimens were collected from the First Affiliated Hospital of Chengdu Medical College (Chengdu, China). Colonoscopy and biopsy were performed for chronic constipation, abdominal pains, distention or occasional bloody mucinous diarrhea. The tissue specimens were surgically removed under endoscopic monitoring. The Institutional Ethics Committee of Chengdu Medical College approved the present study. All patients provided informed consent prior to commencement.

Hematoxylin and eosin $(H \& E)$ staining. The histopathological characteristics of the MC tissue specimens were evaluated using H\&E staining (Beyotime Institute of Biotechnology, Inc., Shanghai, China). The tissues were fixed in $10 \%$ formalin and embedded in paraffin, and then sectioned into $4 \mu \mathrm{m}$ slices prior to staining with $\mathrm{H} \& \mathrm{E}$.

Pathology-specific staining of MC. All grain sizes of the tissue blocks were prepared for specific staining by deparaffinization in xylol and rehydration in serial dilutions of ethanol and distilled water. All the chemical reagents used for specific staining were obtained from Chengdu Changzheng Glass Co., Ltd. (Chengdu, China). The periodic acid Schiff reaction (PAS) was used to detect lipofuscin. Following incubation with $0.5 \%$ periodic acid for $5 \mathrm{~min}$ at room temperature, sections were washed with distilled water for $15 \mathrm{~min}$. Sections were
Table I. Clinical features of patients with melanosis coli.

\begin{tabular}{|c|c|c|}
\hline Clinical feature & Number & Rate $(\%)$ \\
\hline \multicolumn{3}{|l|}{ Gender } \\
\hline Male & 14 & 53.85 \\
\hline Female & 12 & 46.15 \\
\hline \multicolumn{3}{|l|}{ Age (years) } \\
\hline $30-50$ & 6 & 23.07 \\
\hline $51-69$ & 12 & 46.15 \\
\hline$\geq 70$ & 8 & 30.76 \\
\hline \multicolumn{3}{|l|}{ Obstipation } \\
\hline Laxative use & 20 & 76.92 \\
\hline Bloody stools & 3 & 11.53 \\
\hline Abdominal pain & 8 & 30.76 \\
\hline Abdominal distension & 5 & 19.23 \\
\hline Constipation & 12 & 46.15 \\
\hline Dry stool & 12 & 46.15 \\
\hline Loose stools & 3 & 11.53 \\
\hline \multicolumn{3}{|l|}{ Colonoscopic findings } \\
\hline Brown & 18 & 69.23 \\
\hline Red & 8 & 30.76 \\
\hline Mucosal edema & 6 & 23.07 \\
\hline Snake-skin appearance & 7 & 26.92 \\
\hline Neoplasm & 10 & 38.46 \\
\hline Adenocarcinoma & 9 & 34.62 \\
\hline
\end{tabular}

incubated with Schiff's reagent (Sigma-Aldrich, St. Louis, MO, USA) for $30 \mathrm{~min}$ at room temperature, followed by washing under running tap water for $5 \mathrm{~min}$. All sections were counterstained with hematoxylin for $3 \mathrm{~min}$. Masson-Fontana ammoniacal silver staining was used for melanin analysis. The sections were incubated with ammoniacal silver solution (a few drops of ammonia were added into 5\% silver nitrate solution until the precipitation disappeared) in the dark for $15 \mathrm{~min}$ at room temperature and then washed twice with distilled water. The sections were then incubated with $0.2 \%$ gold chloride for $2 \mathrm{~min}$ at room temperature and subsequently washed with distilled water. Sections were then fixed in $2 \%$ sodium thiosulfate and finally counterstained in neutral red for $1 \mathrm{~min}$. Bilirubin is oxidized to biliverdin in an acid medium, and this oxidation reaction occurs rapidly by ferric chloride in trichloroacetic acid solution (16). Sections were incubated with freshly prepared Fouchet's solution $\left(1 \% \mathrm{FeCl}_{3}, 25 \%\right.$ $\mathrm{CCl} 3 \mathrm{COOH}$ ) for $5 \mathrm{~min}$ at room temperature. Sections were washed with distilled water and stained with Van Gieson's solution [1\% fuchsin acid: $1.22 \%$ picric acid (1:9)] for $5 \mathrm{~min}$ at room temperature. Tissue hemosiderin was detected using Prussian blue staining for ferric ion. Sections were incubated with a freshly prepared solution of a 1:1 mixture of $2 \%$ potassium ferrocyanide and $2 \%$ hydrochloric acid for $20 \mathrm{~min}$ at $60^{\circ} \mathrm{C}$. After washing with distilled water, the sections were counterstained in neutral red solution.

Immunohistochemistry. Staining was performed using an Histostain Plus kit (Zhongshan Golden Bridge, Co., Ltd., 
Beijing, China). The specimens were stained with mouse monoclonal immunoglobulin $\mathrm{G}$ (IgG) anti-melanoma antibody (cat. no. ZM0187; Zhongshan Golden Bridge Co., Ltd.). The antibody was diluted at 1:200 and incubated for $30 \mathrm{~min}$ at room temperature.

Terminal deoxynucleotidyl transferase dUTP nick-end labeling (TUNEL) assay. Cell apoptosis in the MC tissues was detected using a TUNEL assay kit (KeyGen Biotech Co., Ltd., Nanjing, China) following the manufacturer's instructions. Briefly, slides containing the tissue sections were incubated with $20 \mu \mathrm{g} / \mathrm{ml}$ proteinase K solution (Sigma-Aldrich) for $30 \mathrm{~min}$ at room temperature. Endogenous peroxidases were inactivated by immersing the slides in $0.3 \%$ hydrogen peroxide in phosphate-buffered saline (PBS; $137 \mathrm{mmol} / 1 \mathrm{NaCl}, 2.7 \mathrm{mmol} / 1$ $\mathrm{KCl}, 10 \mathrm{mmol} / 1 \mathrm{Na}_{2} \mathrm{HPO}_{4}$ and $2 \mathrm{mmol} / 1 \mathrm{KH}_{2} \mathrm{PO}_{4}$ ). A reaction mixture of rTdT (2\% Biotin-11-dUTP and 5\% TdT enzyme in PBS) was added to the slides, and the sections were incubated at $37^{\circ} \mathrm{C}$ for $60 \mathrm{~min}$ to allow the end-labeling reaction to occur. The sections were then incubated with streptavidin-fluorescein isothiocyanate (FITC) solution (1:20 dilution) for $30 \mathrm{~min}$ at room temperature. After washing with PBS three times, the sections were incubated with peroxidase-conjugated antiFITC solution (1:10 dilution) for $30 \mathrm{~min}$ at room temperature. Diaminobenzidine was then added for chromogenesis, for detecting the appearance of a light brown background.

Expression microarray analysis. Total RNA was extracted from the colon MC and normal colon tissues using TRIzol reagent (Gibco Life Technologies, Carlsbad, CA, USA) according to the manufacturer's instructions. The RNA quantity and quality were measured using a NanoDrop ND-1000 spectrophotometer (Thermo Fisher Scientific, Waltham, MA, USA). RNA integrity was assessed using standard denaturing agarose gel electrophoresis. Sample labeling and array hybridization were performed, according to the Agilent One-Color Microarray-based gene expression analysis protocol (Agilent Technologies, Inc., Santa Clara, CA, USA). Briefly, total RNA from each sample was linearly amplified and labeled with Cy3-UTP. The Labeled cRNAs were purified by RNeasy Mini kit (Qiagen $\mathrm{GmbH}$, Hilden, Germany). The concentration and specific activity of the labeled cRNAs (pmol Cy3/ $\mu \mathrm{g}$ cRNA) were measured using the NanoDrop ND-1000. $1 \mu \mathrm{g}$ each labeled cRNA was fragmented by adding $11 \mu \mathrm{l} 10 \mathrm{X}$ blocking agent (Takara Bio, Inc., Shiga, Japan and 2.2 $\mu 1$ 25X fragmentation buffer (Takara Bio, Inc.), then heated at $60^{\circ} \mathrm{C}$ for $30 \mathrm{~min}$. Finally, $55 \mu 12 \mathrm{X}$ gene expression hybridization buffer (Takara Bio, Inc.) was added to dilute the labeled cRNA. Hybridization solution $(100 \mu \mathrm{l})$ was dispensed into the gasket slide and assembled to the gene expression microarray slide, and the slides were incubated for $17 \mathrm{~h}$ at $65^{\circ} \mathrm{C}$. The hybridized arrays were washed with gene expression wash buffer, fixed and scanned using the G2505C Agilent DNA Microarray Scanner (Agilent Technologies, Inc.). Agilent feature extraction software (version 11.0.1.1; Agilent Technologies, Inc.) was used to analyze the acquired array images. Differentially expressed genes with statistical significance were identified through volcano plot filtering. Hierarchical clustering was performed using Agilent Genespring GX software (version 11.5.1; Agilent Technologies, Inc.).
Gene ontology (GO) and pathway analysis were performed according to the standard enrichment computation method (17). The GO project provides a controlled vocabulary to describe gene and gene product attributes in any organism. The ontology covers three domains: i) Biological process; ii) cellular components; and iii) molecular function. Fisher's exact test establishes whether there is more overlap between the differentially expressed list and the $\mathrm{GO}$ annotation list, than would be expected by chance. The P-value denotes the significance of GO term's enrichment in the differentially expressed genes. The lower the $\mathrm{P}$-value, the more significant the GO term $(\mathrm{P}<0.05)$. Pathway analysis is a type of functional analysis that maps genes to Kyoto Encyclopedia of Genes and Genomes (KEGG) pathways (http://www.genome.jp/kegg/pathway.html). The P-value denotes the significance of the pathway correlated with the conditions. When the $\mathrm{P}$-value is lower the pathway is more significant is $(\mathrm{P}<0.05)$.

Western blotting. The samples $(60 \mu \mathrm{g})$ were electrophoresed on $10 \%$ SDS-PAGE gels (Bio-Rad Laboratories, Inc., Hercules, CA, USA) and transferred onto polyvinylidene difluoride membranes (Merck Millipore, Darmstadt, Germany). The rabbit anti-human CYP3A4 polyclonal antibody (cat. no. bs-1472R; Beijing Biosynthesis Biotechnology Co., Ltd., Beijing, China) was diluted at 1:500, added to the membranes and incubated for $2 \mathrm{~h}$ at room temperature. Subsequently, the membranes were incubated with horseradish peroxidase-conjugated IgG (Zhongshan Golden Bridge Co., Ltd.) and analyzed using an enhanced chemiluminescence system (Amersham Pharmacia Biotech, Uppsala, Sweden).

Immunofluorescence assay. Briefly, the blocked tissue sections were incubated overnight at $4^{\circ} \mathrm{C}$ with rabbit anti-human CYP3A4 polyclonal antibody (cat. no. bs-1472R; Beijing Biosynthesis Biotechnology Co., Ltd.). The sections were then incubated with Dylight 649-conjugated secondary antibodies (GeneTex, Inc., San Antonio, TX, USA) for $30 \mathrm{~min}$. The slides were visualized under a fluorescence microscope (TI-S; Nikon Corporation, Tokyo, Japan).

Statistical analysis. Statistical analysis of the results in each experiment was determined using one- or two-way analysis of variance analysis using SPSS 16.0 analysis software (SPSS, Inc., Chicago, IL, USA). $\mathrm{P}<0.05$ was considered to indicate a statistically significant difference.

\section{Results}

Endoscopic and histopathological characteristics of MC. Examining the characteristics of $\mathrm{MC}$ revealed colonoscopy characteristics in three representative cases. Severe MC, in which a marked black-brownish pigmentation was apparent in the mucosa of the whole colon; moderate MC, in which diffuse and brown pigmentation was observed throughout the colon; and mild MC, in which the mucosa had a diffusely brownish, snake-skin appearance (Fig. 1A). H\&E staining revealed that the yellow-brown granular pigment was confined to the tunica propria of the mucosa of large mononuclear histiocytes (Fig. 1B). Of the 26 patients who underwent endoscopy, 
A

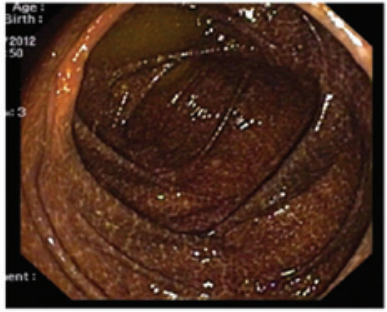

Severe

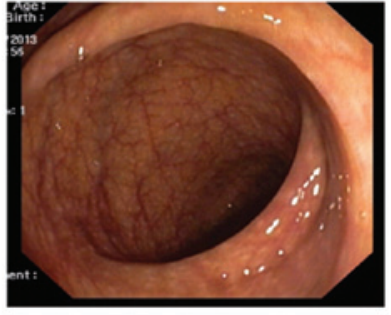

Moderate

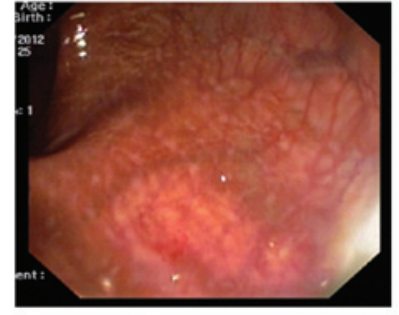

Mild
B

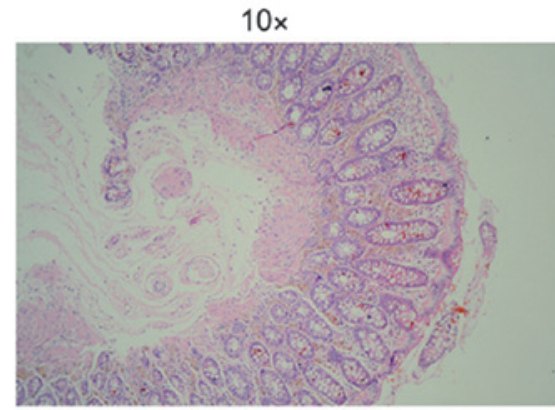

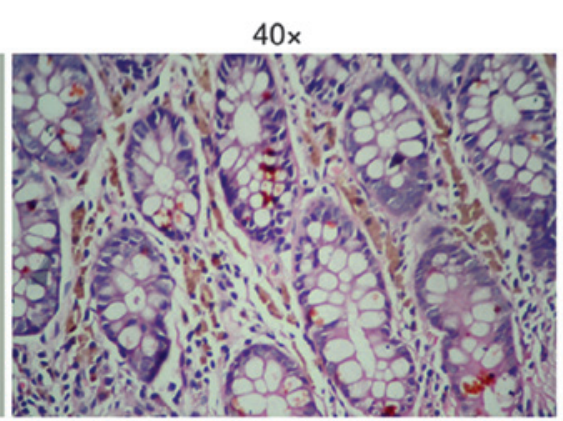

Figure 1. Pigmentation characteristics of melanosis coli. (A) Representative endoscopic images of the three characterized severities. Severe, black-brownish pigmentation of the mucosa of the entire colon. Moderate, diffusely brownish pigmentation. Mild, edematous, snake-skin appearance and pigmentation of the mucosa. (B) Hematoxylin and eosin staining revealing typical pigments in granule-laden macrophages in the lamina propria.

A

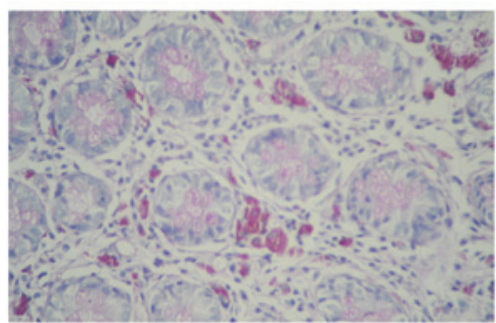

Lipofuscin

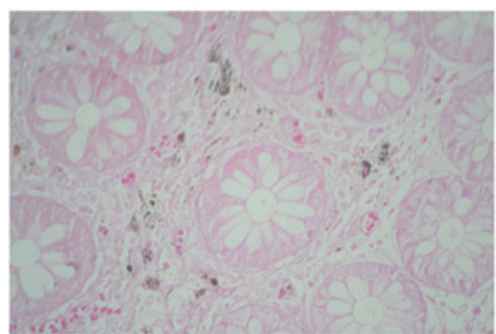

Melanin

B

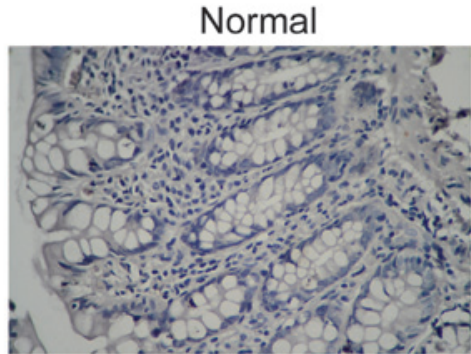

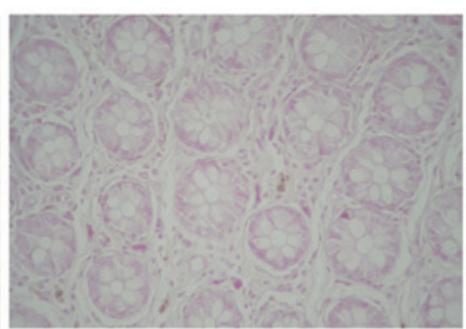

Bile

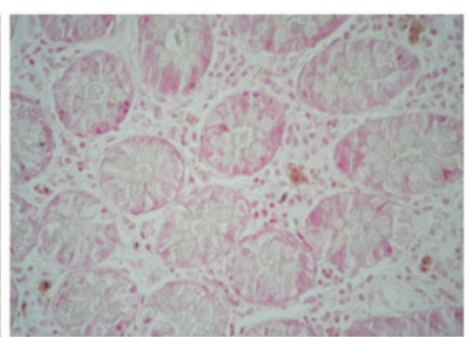

Hemosiderin

$\mathrm{MC}$

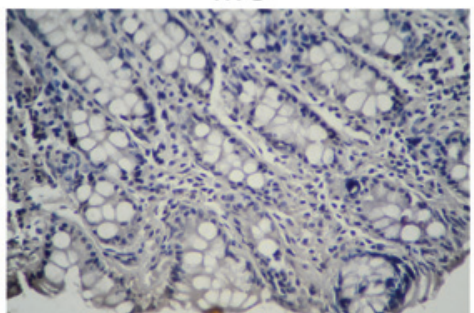

Figure 2. Specific staining and immunohistochemistry for pigment detection in MC tissues. (A) Lipofuscin, periodic acid Schiff reaction demonstrated the presence of lipofuscin, which appears as purple particles. Bile, ferric chloride in trichloroacetic acid medium analysis revealed the absence of bile. Melanin, no obvious black particles were observed following Masson-Fontana ammoniacal silver staining. Hemosiderin, prussian blue staining for ferric ions revealed a negative result for hemosiderin in tissue. Magnification, x40. (B) Paraffin wax-embedded tissues stained using a melanin-antibody. No melanin expression was observed in the MC tissues. Magnification, x40. MC, melanosis coli. 
A

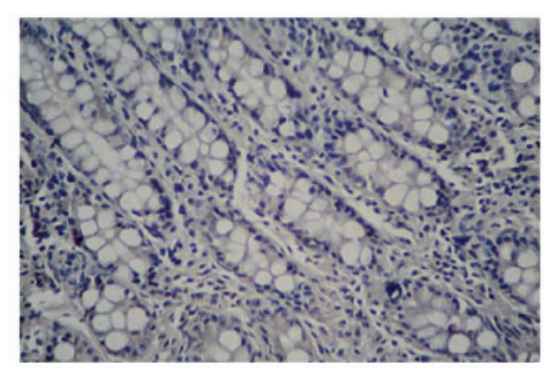

B

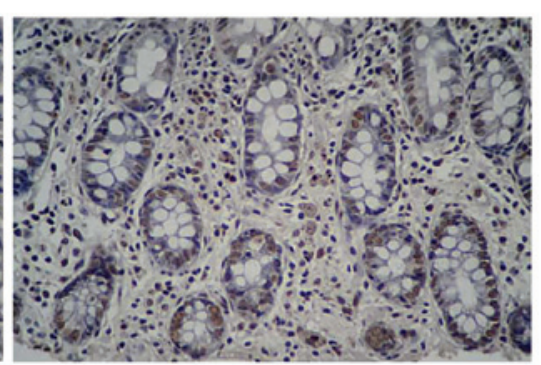

C

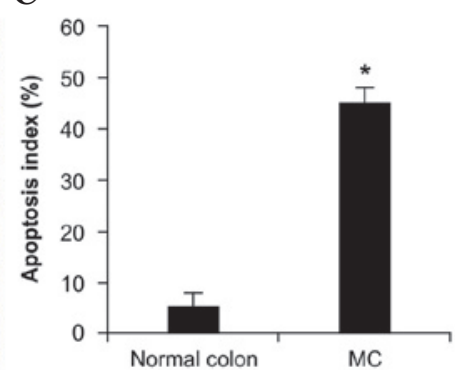

Figure 3. Apoptosis of the colonic cells in MC, determined using a TUNEL assay. (A and B) Sections detected using a TUNEL assay (magnification, x40). (C) Apparent increase in number of apoptotic cells and apoptotic index within the MC tissues, compared with the normal colon tissues. The bar graph indicates the mean \pm standard deviation. ${ }^{*} \mathrm{P}<0.05$, compared with the normal colon tissues. MC, melanosis coli. TUNEL, Terminal deoxynucleotidyl transferase dUTP nick-end labeling.

A

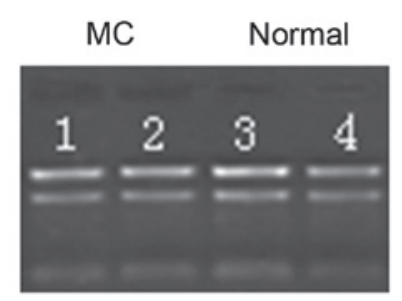

C

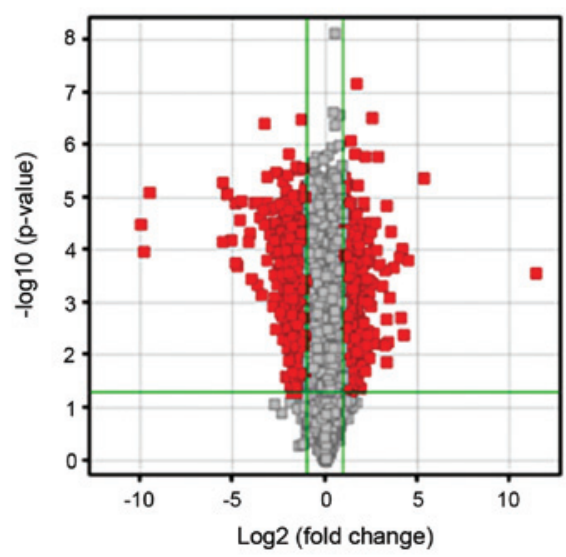

B

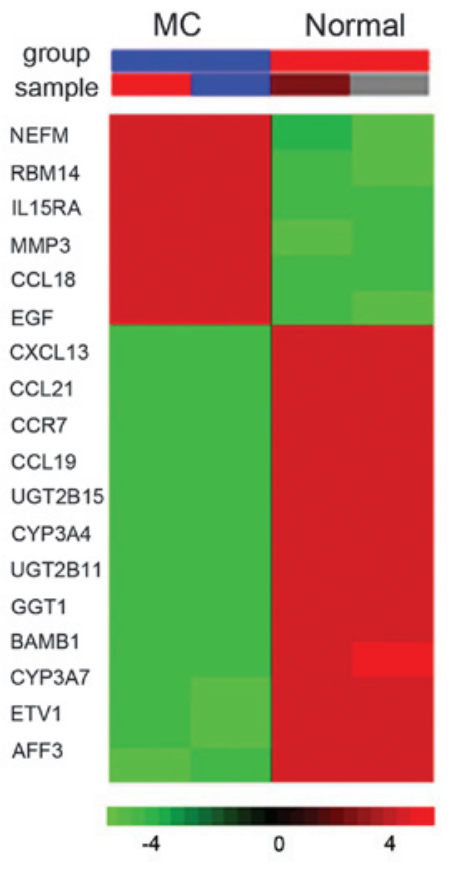

Figure 4. Differentially expressed genes in MC, determined using expression microarray analysis. (A) Total RNA of the samples: Lane 1-2, donors where endoscopic examination revealed MC; lane 3-4, donors where endoscopic examination demonstrated no abnormalities. (B) Heat map for signature genes in each group. Color scale, log2-transformed expression (red, high; green, low) for each gene (row) normalized by the mean of all samples. (C) Volcano plot representation of the differentially expressed genes. The vertical green lines on the left and right correspond to 2.0 -fold downregulation and upregulation, respectively, and the horizontal line indicates a P-value of 0.05 . The red points in the plot indicate the differentially expressed genes between the normal and MC with statistical significance. MC, melanosis coli.

$10(38.46 \%)$ had neoplasia and $10(34.62 \%)$ had adenocarcinoma. It total, $76.92 \%$ of the patients with MC had a history of long-term laxative use (Table I). This suggested that the use of laxatives was associated with MC.

Pigment type in MC. Specific staining and immunohistochemical analyses of the MC tissues indicated that the pigment granules in the lamina propria indicated lipofuscin, but not melanin, bile pigment or hemosiderin. PAS is used to detect the presence of lipofuscin. Light microscopy of the stained sections revealed blue nuclei, a pale red gland cavity and several uniform purple particles in the lamina propria, and there were higher numbers of purple particles in the $\mathrm{MC}$ sections, compared with the normal sections. In addition, no green or blue staining was identified to indicate the presence of bile pigment and hemosiderin, and no obvious black particles were observed in the specimens (Fig. 2A). To confirm the type of pigmentation, $26 \mathrm{MC}$ tissues and 10 normal colon tissues were analyzed using immunohistochemistry. The results indicated that the expression of melanin was absent in the MC and normal colon tissues (Fig. 2B). These results confirmed that the pigment deposits in MC were lipofuscin, not melanin.

Apoptosis in MC tissues. Apoptosis of the colonic cells in MC tissues was evaluated using a TUNEL assay, with which the $26 \mathrm{MC}$ tissues and 10 normal colon specimens were analyzed. Numerous apoptotic cells were observed in the MC tissue sections, and the apoptotic rate was higher than that observed in the normal colon tissue sections. Apoptotic bodies were observed within the macrophages and superficial lamina 


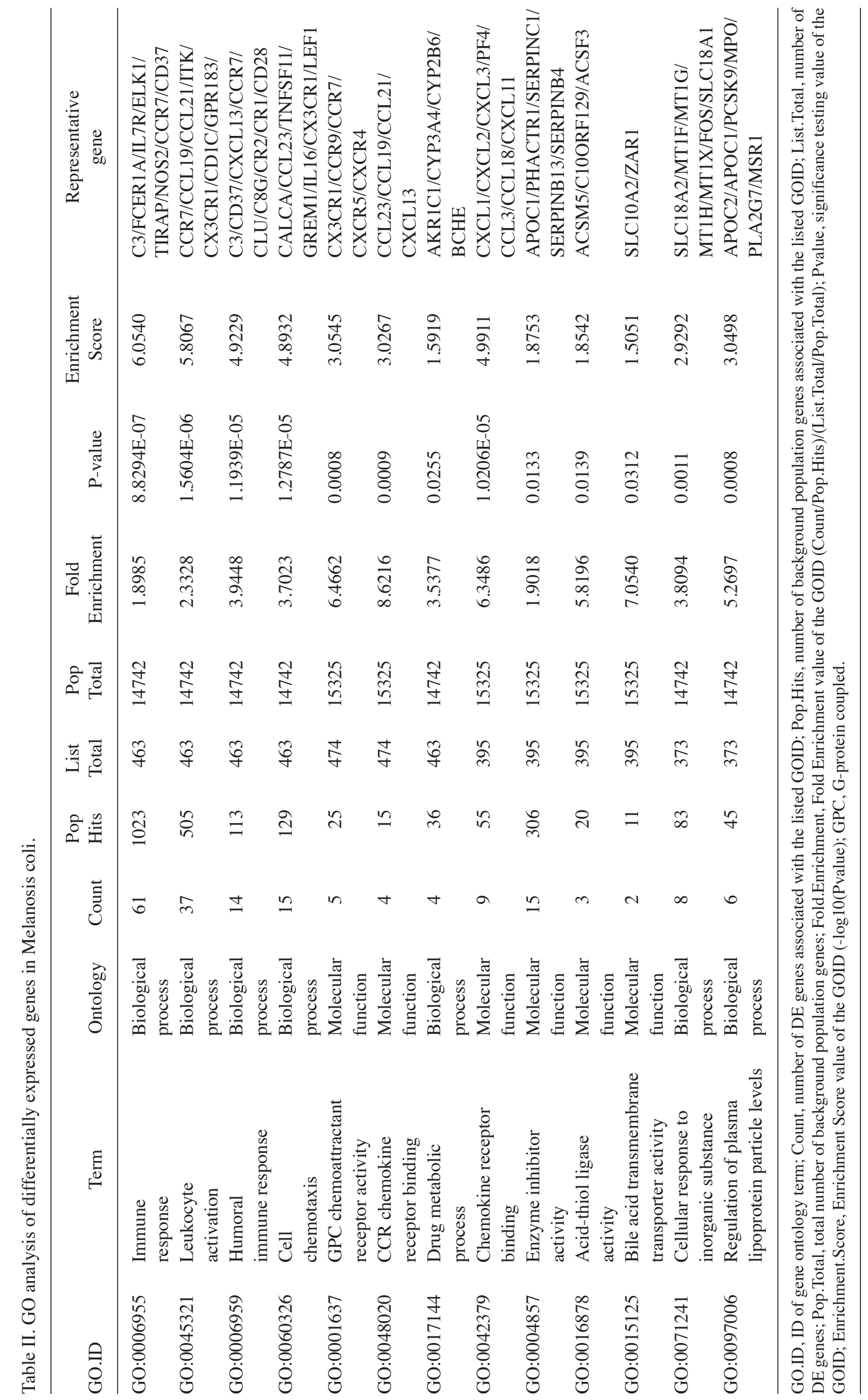


Table III. Top KEGG pathways enriched with downregulated expressed genes and their corresponding Fisher's exact test P-values.

\begin{tabular}{|c|c|c|c|c|}
\hline KEGG pathway name (entry ID) & P-value & $\begin{array}{c}\text { Differentially } \\
\text { expressed genes }\end{array}$ & $\begin{array}{l}\text { Number } \\
\text { of genes }\end{array}$ & Ratio \\
\hline Intestinal immune network for IgA production (hsa04672) & $3.3 \mathrm{E}-05$ & 9 & 50 & 0.180 \\
\hline $\mathrm{NF}-\kappa \mathrm{B}$ signaling pathway (hsa04064) & 4.3E-05 & 12 & 91 & 0.132 \\
\hline Primary immunodeficiency (hsa05340) & 0.0001 & 7 & 36 & 0.194 \\
\hline Cytokine-cytokine receptor interaction (hsa04060) & 0.0002 & 21 & 271 & 0.077 \\
\hline Hematopoietic cell lineage (hsa04640) & 0.0006 & 10 & 88 & 0.114 \\
\hline Vascular smooth muscle contraction (hsa04270) & 0.0013 & 12 & 131 & 0.092 \\
\hline Steroid hormone biosynthesis (hsa00140) & 0.0026 & 7 & 57 & 0.123 \\
\hline Retinol metabolism (hsa00830) & 0.0051 & 7 & 64 & 0.109 \\
\hline Starch and sucrose metabolisms (hsa00500) & 0.0103 & 6 & 56 & 0.107 \\
\hline Metabolism of xenobiotics by cytochrome P450 (hsa00980) & 0.0112 & 7 & 74 & 0.095 \\
\hline Arachidonic acid metabolism (hsa00590) & 0.0191 & 6 & 64 & 0.094 \\
\hline
\end{tabular}

KEGG, Kyoto Encyclopedia of Genes and Genomes. The ratio represents the percentage of differentially expressed genes in the indicated pathway.

Table IV. Top KEGG pathways enriched with upregulated expressed genes and their corresponding Fisher's exact test P-values.

\begin{tabular}{lrrr}
\hline KEGG pathway name (entry ID) & P-value & $\begin{array}{c}\text { Differentially } \\
\text { expressed genes }\end{array}$ & $\begin{array}{c}\text { Number } \\
\text { of genes }\end{array}$ \\
\hline Cytokine-cytokine receptor interaction (hsa04060) & 0.0011 & 17 & 271 \\
Chemokine signaling pathway (hsa04062) & 0.0019 & 13 & 189 \\
Salmonella infection (hsa05132) & 0.0022 & 8 & 86 \\
Mineral absorption (hsa04978) & 0.0024 & 6 & 0.069 \\
Rheumatoid arthritis (hsa05323) & 0.0034 & 8 & 0.093 \\
Butirosin and neomycin biosynthesis (hsa00524) & 0.0069 & 2 & 0.118 \\
Bile secretion (hsa04976) & 0.0133 & 6 & 5 \\
Glycine, serine and threonine metabolism (hsa00260) & 0.0191 & 4 & 0.087 \\
Serotonergic synapse (hsa04726) & 0.0355 & 7 & 0.083 \\
Collecting duct acid secretion (hsa04966) & 0.0361 & 3 & 0.105 \\
Melanoma (hsa05218) & 0.0436 & 5 & 0.061 \\
\hline
\end{tabular}

KEGG, Kyoto Encyclopedia of Genes and Genomes. The ratio represents the percentage of differentially expressed genes in the indicated pathway.

propria of the colonic epithelium (Fig. 3). These results indicates that pigment storage is a consequence of apoptosis in colonic epithelial cells.

Analysis of the gene chip expression profile. Data from three independent samples demonstrated that 1,718 genes were differentially expressed between the MC and control samples (Fig. 4). Of these, 879 genes were downregulated and 739 genes were upregulated, as shown by the Volcano plot representation in Fig. 4C. The most significantly upregulated genes were CCL18, NEFM, EGF and IL15RA, and the most significantly downregulated genes were CYP3A4, CYP3A7, UGT2B11 and UGT2B15 (Fig. 4B). The GO functional class scoring of the differentially expressed genes demonstrated that the most affected categories were as follows: Immune response, lymphocyte activation, humoral immune response, cell chemotaxis, G-protein-coupled chemoattractant receptor activity, CCR chemokine receptor binding and drug catabolic process for the downregulated genes and chemokine receptor binding, enzyme inhibitor activity, acid/thiol ligase activity, bile acid transmembrane transporter activity, cellular response to inorganic substance and lipoprotein particle for the upregulated genes (Table II). Accordingly, the most affected pathways for the downregulated genes were as follows: Immune network, $\mathrm{NF}-\kappa \mathrm{B}$ signaling pathway, metabolism of xenobiotics by cytochrome $\mathrm{P} 450$, vitamin digestion and absorption (Table III) the most affected pathways for the upregulated genes were as follows: Salmonella infection, mineral absorption, bile secretion, collecting duct acid secretion and melanoma (Table IV).

Detection of CYP3A4. P450 families of CYP1, CYP2 and CYP3 are the predominant contributors to the oxidative metabolism of $>90 \%$ of clinical drugs. CYP3A4 is one of 
A
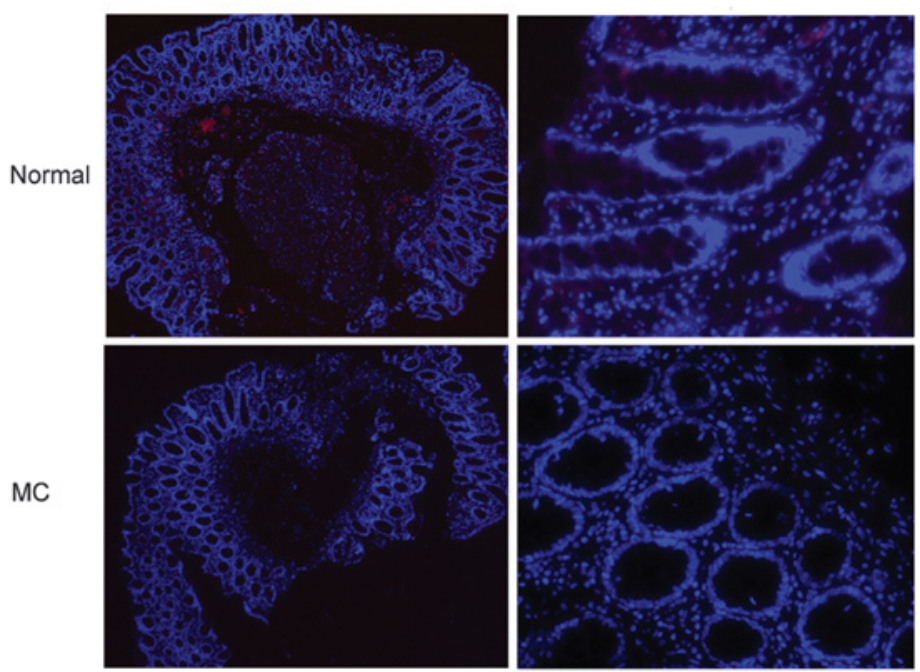

B

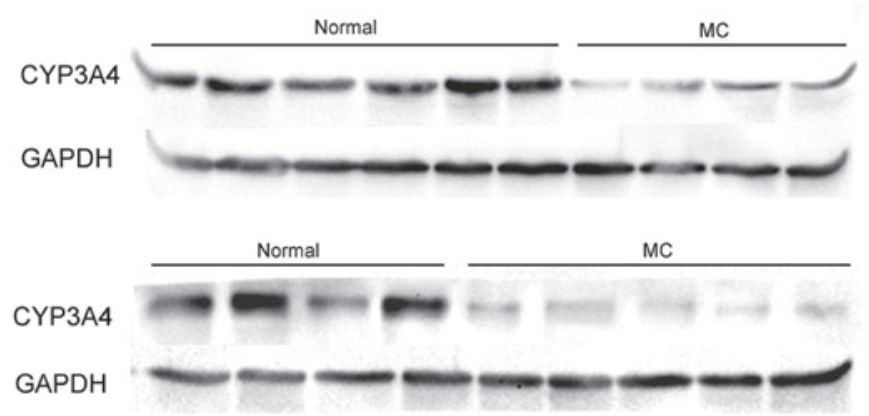

Figure 5. Elevated expression of CYP3A4 in MC and normal tissues. (A) Immunofluorescence assay. Red staining indicates protein expression of CYP3A4, while blue staining indicates the nuclei of the cells. Magnification: Left column, x4; right column, x20. (B) Immunoblot analysis of the expression of CYP3A4. Results are representative of 19 independent sample tissues per group. Expression of CYP3A4 was standardized to that of GAPDH. MC, melanosis coli.

these, and is predominantly present in the intestine. The chip expression data revealed that CYP3A4 was downregulated in MC by 11.0-fold, compared with normal tissue. To further verify the results of the gene chip screening, the expression levels of CYP3A4 were assayed using western blotting and an immunofluorescence assay in the present study. The results indicated that the expression of CYP3A4 in MC was higher than in normal tissue (Fig 5) and were, therefore, in accordance with the results of the gene chip screening.

\section{Discussion}

MC refers to an abnormality in which brown or black pigmentation is deposited in the colonic mucosa. It is a relatively common finding in colonic biopsies and resected specimens, however, the histopathology and pathogenesis of MC remain to be fully elucidated. In the present study, the type of pigment in $\mathrm{MC}$ was investigated by performing specific staining and immunohistochemical analyses in $26 \mathrm{MC}$ specimens. The pigment deposits in MC were observed to contain lipofuscin and not melanin, bile pigments or hemosiderin. This condition, in which pigment deposits consist of lipofuscin rather than melanin is also referred to as pseudo-MC. In addition, there were a higher number of apoptotic cells in MC, compared with normal tissues. Expression microarray analysis demonstrated that the significantly downregulated genes were CYP3A4,
CYP3A7, UGT2B11 and UGT2B15 in MC tissue, and western blotting and immunofluorescence analyses indicated that the expression of CYP3A4 in normal tissue was higher than that in $\mathrm{MC}$.

The pathogenesis of MC has not been investigated in previous studies at depth. Several hypotheses have been suggested to explain pigment formation in MC. For example, it has been suggested that the formation of pigment granules is associated with apoptosis in colonic mucous membrane epithelial cells induced by purgatives (18). It has also been suggested that constipation leads to the retention of protein-rich foods in the intestinal tract, and that the protein degradation products are converted into melanin or lipofuscin, which are phagocytized by macrophages in the lamina propria (8). Despite these hypotheses, there is no clear experimental evidence to support any single pathogenesis for MC. In the present study, TUNEL apoptosis analysis revealed numerous apoptotic bodies in the epithelium and superficial lamina propria in the colonic mucosal biopsies from patients with MC. Pigment storage is a consequence of colonic epithelial cells apoptosis, in which the apoptotic cells are swallowed by macrophages, which migrate in the lamina propria and the conversion into lipofuscin pigment occurs by lysosomal enzymes (19).

The gene chip technique has been widely used to detect gene expression differences using comparative analysis. In the present study, the Agilent gene chip to analyze the gene 
expression profile of human MC and normal colon tissues. As shown in Fig. 4, significant changes were observed in the expression of several genes in MC. These genes included those involved in the intestinal immune network, $\mathrm{NF}-\kappa \mathrm{B}$ signaling pathway and metabolism of xenobiotics and drug by cytochrome P450 and melanoma. The most significantly downregulation genes were CYP3A4, CYP3A7, UGT2B11 and UGT2B15. These genes belong to the cytochrome P450 superfamily, which is involved in the metabolism of xenobiotics and drugs (20). The human CYP superfamily contains 57 functional genes and 58 pseudogenes. Among these, the members from the CYP1, CYP2 and CYP3 families are the predominant contributors to the oxidative metabolism of $>90 \%$ of clinical drugs $(21,22)$, and CYP3A4 is one of these, which is predominantly present in the intestine.

Aloe and emodin are anthraquinones known to be metabolized by P450s. It has been reported that aloe vera juice inhibits CYP3A4 and CYP2D6 irreversibly in vitro, having significantly different half maximal inhibitory concentration values (23), and emodin inhibits $\mathrm{P} 450$ with an antimutagenic effect $(24,25)$. These biological effects of emodin prompted the present study to investigate anthraquinones as potential P450 inhibitors. The chip expression data in the present study demonstrated that CYP3A4 was downregulated in the MC tissues by 11.0 -fold, compared with normal tissues. Western blotting and immunofluorescence assays also indicated that the expression of CYP3A4 in the MC tissue was lower than in the normal tissue (Fig. 5).

The data of the present study demonstrated that the pigment deposits in MC contain lipofuscin, and do not contain melanin, bile pigment or hemosiderin, and numerous apoptotic bodies were observed in the epithelium and superficial lamina propria in the colonic mucosal biopsies. Expression microarray analysis revealed that the $\mathrm{P} 450$-associated genes were significantly downregulated in MC tissues, and further experiments confirmed that the expression of CYP3A4 in the normal tissue was higher than in the MC tissue. To the best of our knowledge, this is the first time to demonstrate that, for MC patients, long time use of anthraquinone laxatives may inhibit P450, particularly CYP3A4, in the intestine. These findings increase understanding for assistance in further investigations of MC.

\section{Acknowledgements}

This study was supported by the National Natural Science Foundation of China (grant no. 81302170), the Natural Science Foundation of Chengdu Medical College (grant no. CYZ12-005) and the National Clinical Medicine Research Foundation of China (grant no. L2012055).

\section{References}

1. Li D, Browne LW and Ladabaum U: Melanosis coli. Clin Gastroenterol Hepatol 7: A20, 2009.
2. Kunkel J, Schmidt S, Loddenkemper C, Zeitz M and Schulzke JD: Chronic diarrhea and melanosis coli caused by wellness drink. Int J Colorectal Dis 24: 595-596, 2009.

3. Samenius B: The clinical importance of melanosis coli. Proc R Soc Med 52 (Suppl): 105-106, 1959.

4. Pardi DS, Tremaine WJ, Rothenberg HJ and Batts KP: Melanosis coli in inflammatory bowel disease. J Clin Gastroenterol 26: 167-170, 1998.

5. Chen JY, Pan F, Zhang T, Xia J and Li YJ: Experimental study on the molecular mechanism of anthraquinone cathartics in inducing melanosis coli. Chin J Integr Med 17: 525-530, 2011.

6. Chen JY, Pan F and Zhang T: Rhubarb induced change of tumor necrosis factor-alpha level in guinea pig model of melanosis coli and its significance. Zhongguo Zhong Xi Yi Jie He Za Zhi 29: 140-142, 2009 (In Chinese).

7. Wang T, Chen ZW and Streutker CJ: Melanosis coli sparing adenomatous polyps: Novel findings using cleaved caspase-3 immunohistochemistry. Histopathology 62: 819-821, 2013.

8. Pearce CB, Martin H, Duncan HD, Goggin PM and Poller DN: Colonic lymphoid hyperplasia in melanosis coli. Arch Pathol Lab Med 125: 1110-1112, 2001.

9. Loveday RL, Hughes MA, Lovel JA, and Duthie GS: Melanosis coli in the absence of anthranoid laxative use harbouring adenoma. Colorectal Dis 15: 1044-1045, 2013.

10. Franken FH and Wiechers B: [Melanosis coli(author's transl)]. Leber Magen Darm 5: 269-271, 1975 (In German).

11. Park C, Cho NH and Jeong HJ: Melanosis coli - histochemical and immunohistochemical comparison of the pigments of melanosis coli and Dubin-Johnson syndrome. Yonsei Med J 31: 27-32, 1990.

12. Benavides SH, Morgante PE, Monserrat AJ, Zárate J and Porta EA: The pigment of melanosis coli: A lectin histochemical study. Gastrointest Endosc 46: 131-138, 1997.

13. Allen EA, Ali SZ and Erozan YS: Pigment-laden macrophages in ascitic fluid associated with melanosis coli. Acta Cytol 41: 1249-1251, 1997.

14. Tsobanidou Ch: Melanosis coli in two patients with colorectal neoplasia. J BUON 10: 131-133, 2005.

15. Puppa G and Colombari R: Brown colon (melanosis coli) harbouring pale tumors (adenocarcinoma and an adenomatous polyp). J Gastrointestin Liver Dis 18: 509-511, 2009.

16. Onks DL, Robertson AF and Brodersen R: The effect of chloral hydrate and its metabolites, trichloroethanol and trichloroacetic acid, on bilirubin-albumin binding. Pharmacol Toxicol 71: 196-197, 1992.

17. Tian L, Greenberg SA, Kong SW, Altschuler J, Kohane IS and Park PJ: Discovering statistically significant pathways in expression profiling studies. Proc Natl Acad Sci USA 102: 13544-13549, 2005.

18. Walker NI, Bennett RE and Axelsen RA: Melanosis coli. A consequence of anthraquinone-induced apoptosis of colonic epithelial cells. Am J Pathol 131: 465-476, 1988.

19. Chen JY, Pan F, Zhang T, Xia J and Li YJ: Experimental study on the molecular mechanism of anthraquinone cathartics in inducing melanosis coli. Chin J Integr Med 17: 525-530, 2011.

20. Pandey AV and Flück CE: NADPH P450 oxidoreductase: structure, function, and pathology of diseases. Pharmacol Ther 138: 229-254, 2013.

21. Nebert DW and Russell DW: Clinical importance of the cytochromes P450. Lancet 360: 1155-1162, 2002.

22. Guengerich FP: Cytochrome P450s and other enzymes in drug metabolism and toxicity. AAPS J 8: E101-E111, 2006.

23. Djuv A: Co-use of drugs and herbal remedies in general practice and in vitro inhibition of CYP3A4, CYP2D6 and P-glycoprotein by the common herb aloe vera (Doctoral thesis). Department of Cancer and Molecular Medicine, Faculty of Medicine, Norwegian University of Science and Technology, 333: 1503-8181, 2013 (In Norwegian).

24. Sridhar J, Liu J, Foroozesh M and Klein Stevens CL: Inhibition of cytochrome p450 enzymes by quinones and anthraquinones. Chem Res Toxicol 25: 357-365, 2012.

25. Takahashi E, Fujita K, Kamataki T, Arimoto-Kobayashi S, Okamoto K and Negishi T: Inhibition of human cytochrome P450 1B1, 1A1 and 1A2 by antigenotoxic compounds, purpurin and alizarin. Mutat Res 508: 147-156, 2002. 